candidates for involvement in functional chromatin and nuclear organization, we investigated the actin family, which consists of conventional actin and actin-related proteins (Arps). Interestingly, a portion of actin and some Arp subfamilies are localized in the cell nucleus. Monomeric G-actin and the nuclear Arps are known to contribute to genome functions (including transcription and DNA damage repair) as components of chromatin remodeling complexes and histone modification complexes. In addition to nuclear G-actin, nuclear actin filament (F-actin) is also involved in genome functions and nuclear organization. Although a sufficient amount of actin is found in the nucleus, information regarding factors involved in regulating the formation of nuclear F-actin is still limited. We showed that Arp4 (one of the nuclear Arps) is a suppressor of nuclear F-actin formation, which suggests that crosstalk between actin family proteins in the nucleus performs important roles in chromatin functions and nuclear organization. For further analysis and operation of nuclear actin family proteins, we screened and evaluated bicyclic peptides binding to these molecules. Bicyclic peptides contain two macrocyclic rings, and this structure contributes to high affinity binding to target molecules. We performed screening of bicyclic peptide libraries by the phage display technique, and obtained bicyclic peptides for nuclear Arps and G-actin. We introduced these bicyclic peptides into living cells by electroporation, and evaluated the peptides. Bicyclic peptides for Arp5 and Arp8 (components of the INO80 chromatin remodeling complex) inhibited functions of INO80 complex in the living cells. We also successfully delivered bicyclic peptides for G-actin into the nucleus by tagging a nuclear localization signal (NLS). We observed that the NLS-bicyclic peptides for G-actin suppressed nuclear F-actin formation and impaired the function of nuclear F-actin in DNA damage repair. These bicyclic peptides provide novel information on the roles of actin family proteins in the nucleus.

doi: http://dx.doi.org/10.7124/bc.0009B3

\section{Nuclear lipid Islets as integrators of Polymerase II transcription and pre-mRNA processing}

\author{
Martin Sztacho 1 , Pavel Hozak ${ }^{1,2,3}$ \\ ${ }^{1}$ Institute of Molecular Genetics ASCR v.v.i., De- \\ partment of Biology of the Cell Nucleus, Vídeňská \\ 1083, 142 20, Prague 4, Czech Republic; ${ }^{2}$ Micros- \\ copy Centre, Institute of Molecular Genetics ASCR \\ v.v.i., Vídeňská 1083, 142 20, Prague 4, Czech \\ Republic; ${ }^{3}$ Institute of Molecular Genetics ASCR \\ v.v.i., Division BIOCEV, Laboratory of Epigenetics \\ of the Cell Nucleus, Průmyslová 595, 252 50, \\ Vestec, Czech Republic \\ martin.sztacho@img.cas.cz
}

The eukaryotic nuclear environment possess a dynamic highly organized architecture. Processes such as gene expression, DNA repair or RNA processing occur often in membraneless compartments. These structures constitute of e.g. nucleic acids, proteins, lipid multimolecular condensates. Our laboratory recently discovered nanoscale globular $(\sim 100 \mathrm{~nm})$ Nuclear Lipid Islets (NLIs) structures containing PI(4,5)P2 (PIP2), which are involved in efficient RNAPII transcription [1]. We aim to describe the possible function of PIP2containing NLIs in integrating RNAPII tran- 
scription and pre-mRNA splicing at their surface. Methods: To decipher whether PIP2containing NLIs surface recruit a regulatory proteins abovementioned processes, we employed a comparative quantitative mass spectrometry (MS) analysis of PIP2 nuclear fraction combined with super-resolution microscopy visualization of candidate proteins. Results: Our quantitative MS approach identified more than 300 putative NLIs proteins, which represents $35 \%$ of nuclear PIP2 - associated proteins. Moreover, $50 \%$ of those proteins are connected to gene expression and more that 30 $\%$ to RNA processing. Super-resolution microscopy showed that candidate proteins form foci in nucleoplasm and associate with sub-population of NLIs. In contrary, proteins predicted by MS as NLIs-nonassociated showed random distribution in respect to nucleoplasmic PIP2 foci. Conclusions: It is known that RNA splicing occurs co-transcriptionally. We show that sub-population of NLIs interact with regulators of both RNAPII transcription and pre-mRNA splicing [2]. We hypothesis that the surface of NLIs facilitate spatiotemporal regulation of these processes.

This study was supported by the Czech Academy of Sciences (JSPS-18-18); Grant Agency of the Czech Republic (16-03346S, 17-09103S, 15-08738S); Technology Agency of the Czech Republic (TE01020118); IMG ASCR, v. v. i.(RVO: 68378050); European Regional Development Fund (CZ.02.1.01/0.0/0.0/16 013/0001775 ). Microscopy Centre - IMG AS CR the Czech-Biolmaging project (LM2015062 funded by MEYS CR).

References: 1. Sobol, M., et al., Nuclear phosphatidylinositol 4,5-bisphosphate islets contribute to efficient RNA polymerase II-dependent transcription. J Cell Sci, 2018. 131(8). 2. Sztacho, M., et al., Nuclear phosphoinositides and phase separation: Important players in nuclear compartmentalization. Adv Biol Regul, 2018. doi: http://dx.doi.org/10.7124/bc.0009B4

\section{Nuclear Vinculin involvement in mouse spermatogenesis}

Petr Flachs $^{1}$, Alzbeta Darasova ${ }^{1}$, Pavel Hozak ${ }^{1,2}$

${ }^{1}$ Laboratory of Epigenetics of the Cell Nucleus, Institute of Molecular Genetics of the ASCR, division BIOCEV, Prague, Czech Republic; ${ }^{2}$ Laboratory of Biology of the Cell Nucleus, Institute of Molecular Genetics of the ASCR, Prague, Czech Republic petr.flachs@img.cas.cz

The focal adhesion protein vinculin (VCL) is a cytoskeletal protein associated with cell-matrix junctions where it acts as a molecular clutch between the actin cytoskeleton and the extracellular matrix via talins and integrins. However, our study reveals new localization and unexpected role of VCL in the nuclei of spermatocytes during Prophase I. In particular, VCL localizes in the nuclear interior along the meiosisspecific structure synaptonemal complex (SC) and in the centromeric regions of homologous chromosomes. To understand the role of VCL in the early meiotic division, we prepared a VCL conditional knock-out mouse model (VCL cKO) where the VCL deletion is manifested explicitly during the early stages of spermatogenesis. In comparison with the wild-type control, our mouse model shows significantly decreased ability of males to sire offspring together with increased percentage of malformed sperm. According to our short-term cultivation experiments primary spermatocytes of VCL cKO males exhibit deficiency in progression throughout late prophase I. Likewise, FACS (fluorescence-activated cell sorting) analysis revealed the enrichment of the prophase I spermatocytes. The chromosomal pairing and the crossing-over formation appear intact. However, spermato- 